\title{
ВЛИЯНИЕ ДИНАМИЧЕСКОГО НАГРУЖЕНИЯ НА ЭНЕРГОПОГЛОЩЕНИЕ В ПРОЦЕССАХ ХОЛОДНОГО ПЛАСТИЧЕСКОГО ДЕФОРМИРОВАНИЯ
}

В условиях эксплуатации сталей повышенной прочности с высокой энергопоглощающей способностью очевидна необходимость оценки качества изделий, их деформационной способности поглощать энергию при ударной нагрузке твердым индентором. В работе представлен подход к решению этой проблемы.

Цель работы - разработать феноменологический критерий и создать метод, позволяющий оценить энергию формоизменения, затраченную на внедрение цилиндрического пуансона в пластическое полупространство.

Постановка задачи - в стальную пластину толщиной $h$ внедряется твердый индентор с изменяющейся скоростью от $v_{0}$ до $v_{1}$. Силу сопротивления принимаем пропорциональной скорости:

$$
R=k \dot{x}
$$

Рассмотрим алгоритм расчета энергетических затрат на проникновение пуансона в пластически деформируемую среду. Следуя работе [1], в которой разработан феноменологический подход для определения энергии деформации, затраченной на деформацию и разрушение транспортных средств, удельную потенциальную энергию определяют интегрированием функции упрочнения металлов, где верхний предел интеграла может быть определен экспериментально. Используем этот подход к решению задачи для определения коэффициента сопротивления среды при проникновении в нее твердого пуансона.

Формируем карту материала, учитывающую изотропное упрочнение, которая включает кривую течения в координатах «интенсивность напряжений $\sigma_{u},-$ интенсивность деформаций $\varepsilon_{u}$ » и диаграмму пластичности в координатах «предельная степень накопленной интенсивности деформаций к моменту разрушения $\varepsilon_{p}$ - показатель напряженного состояния $\eta$ ».

Кривая течения, не зависящая от вида напряженного состояния в координатах $\sigma_{u}-$ интенсивность напряжений:

$$
\sigma_{u}=\frac{1}{\sqrt{2}} \sqrt{\left(\sigma_{1}-\sigma_{2}\right)^{2}+\left(\sigma_{2}-\sigma_{3}\right)^{2}+\left(\sigma_{1}-\sigma_{3}\right)^{2}}
$$

и $\varepsilon_{u}-$ интенсивность деформаций:

$$
\varepsilon_{u}=\frac{\sqrt{2}}{3} \sqrt{\left(\varepsilon_{1}-\varepsilon_{2}\right)^{2}+\left(\varepsilon_{2}-\varepsilon_{3}\right)^{2}+\left(\varepsilon_{1}-\varepsilon_{3}\right)^{2}},
$$

где $\sigma_{1}, \sigma_{2}, \sigma_{3}$ - главные напряжения, $\varepsilon_{1}, \varepsilon_{2}, \varepsilon_{3}$ - главные деформации.

$\mathrm{C}$ целью использования кривой течения в расчетах, ее представили в виде степенной функции:

$$
\sigma_{u}=A \varepsilon_{u}^{n}
$$

где $A$ и $n$ - коэффициенты аппроксимации, имеющие физический смысл. 
Для изотропного материала коэффициент $A$ - это интенсивность напряжений при интенсивности деформаций $\varepsilon_{u}=1$. Показатель степени $n$ в формуле (1) - это интенсивность деформаций при максимальной нагрузке на условной диаграмме растяжения.

В дальнейшем рассмотрим движение пуансона как в статических условиях, так и в условиях ударного нагружения. При динамических нагрузках скорость деформаций оказывает влияние на способность материалов к упрочнению. Используя модель материала, чувствительную к различным скоростям деформирования, приведем формулу, аппроксимирующую кривую течения в условиях проявления скоростного эффекта [1]:

$$
\sigma_{u}=\mathrm{A}_{v} \varepsilon_{u}^{n_{v}}
$$

где коэффициенты $\mathrm{A}_{v}$ и $n_{v}$ принимают значения, соответственно равные:

$$
\begin{aligned}
& A_{v}=A\left[1,045+\frac{\ln \left(0,0027+\dot{\varepsilon}_{u}\right)}{135}\right] \\
& n_{v}=n \exp \left[-0,1273 \ln \left(1+\dot{\varepsilon}_{u}\right)\right] .
\end{aligned}
$$

В формулах (2), (3) и (4) $\mathrm{A}_{v}$ - коэффициент аппроксимации кривой течения, учитывающий влияние скорости деформирования, $\dot{\varepsilon}_{u}-$ скорость интенсивности деформаций, $n_{v}-$ показатель степени, учитывающий влияние скорости деформирования.

Удельную потенциальную энергию деформирования получим интегрированием выражения:

$$
W_{y \partial}=\int_{0}^{\varepsilon_{u}^{*}} \sigma_{u} d \varepsilon_{u}
$$

После подстановки (2) в (5), получим:

$$
W_{y \partial}=\int_{0}^{\varepsilon_{u}^{*}} A_{v} \varepsilon_{u}{ }^{n_{v}} d \varepsilon_{u}=A_{v} \int_{0}^{\varepsilon_{u}^{*}} \varepsilon_{u}^{n_{v}} d \varepsilon_{u}=\frac{A_{v} \varepsilon_{u}{ }^{n_{v}+1}}{n_{v}+1} .
$$

Энергия деформации определяется по формуле:

$$
W_{\text {def }}=W_{y \partial} V_{\text {def }},
$$

где $V_{d e f}$ - объем, охваченный пластической деформацией, определяется как объем цилиндра с текущими диаметром $d_{i}$ и высотой $h_{i}$.

Тогда:

$$
W_{d e f}=W_{y \partial} \frac{\pi d_{i}^{2}}{4} h_{i}
$$

Учитывая, что:

$$
R=k \dot{x}=\frac{W_{d e f}}{h_{i}}
$$

коэффициент сопротивления $k$ примет значение, равное: 


$$
k=\frac{W_{d e f}}{h_{i} \dot{x}}=\frac{W_{d e f}}{h_{i} v_{d e f}},
$$

где $v_{d e f}$ - скорость прохождения индентора в упрочняющейся среде от $v_{0}$ до $v_{1}$.

Изменение скорости движения пуансона в упрочняющейся среде определим по формуле:

$$
v_{d e f}=v_{0} \exp \left(\frac{-h_{i}}{h_{0}} \ln \frac{v_{0}}{v_{1}}\right) .
$$

Приведем пример расчета энергетических затрат при проникновении пуансона сквозь пластину толщиной $h=5,5$ мм из мартенситной стали, для которой учтены следующие механические характеристики [2]: $\sigma_{b}=1700 \mathrm{MПа,} \sigma_{0,2}=1550 \mathrm{MПа,} \mathrm{показатель} \mathrm{степени} \mathrm{в} \mathrm{формуле}$ (1) $n=0,23$, коэффициент упрочнения $A=2893$ МПа, скорость пуансона $v_{0}=700 \mathrm{~m} / \mathrm{c}$, $\dot{\varepsilon}_{\mathrm{u}}=7825 \mathrm{c}^{-1}$.

Расчет коэффициентов $\mathrm{A}_{v}$ и $n_{v}$ по формулам (3) и (4) соответственно дает значения коэффициентов аппроксимации $\mathrm{A}_{v}=3215$ МПа и $n_{v}=0,0783$.

В статических условиях при деформации, например, $\varepsilon_{u}=0,6$, интенсивность напряжений $\sigma_{u}=2572$ МПа, а в динамических $\sigma_{u}=3089$ МПа.

Следовательно, в динамических условиях при $\varepsilon_{u}=0,6, \sigma_{u}$ возрастает в 1,2 раза, а удельная потенциальная энергия ( $\left.W_{y \partial}\right)$ в 1,15 раза.

Объем, охваченный пластической деформацией, принимаем равным объёму внедренного в деформированный металл пуансона с учетом объема упрочненной зоны (пластической волны).

Следуя работе [3], при внедрении сферического наконечника в пластическую среду диаметр пластической волны $d_{i}$ составляет $1.5 d_{0}$. В нашем случае для $d_{0}=7.62$ мм, диаметр $d_{i}=1,143 \mathrm{~cm}$.

При вычислении удельной энергии по формуле (6) необходимо знать величину деформации, при которой произошло разрушение металла в условиях сдвига с наложением гидростатического давления.

В работе [4] приведены экспериментальные результаты оценки предельных деформаций, зависящих от безразмерных показателей напряженного состояния:

$$
\eta=\frac{\sigma_{1}+\sigma_{2}+\sigma_{3}}{\sigma_{u}}
$$

При внедрении индентора в пластическую среду показатель $\eta$ в момент разрушения равен $\eta=-1,73$ [4]. Используя аппроксимацию диаграмм пластичности, приведенную в работе [4]:

$$
\varepsilon_{p}=\varepsilon_{p}(\eta=0) \exp \left(-\lambda_{i} \cdot \eta\right)
$$

где $\varepsilon_{p}(\eta=0)-$ предельная деформация при сдвиге; $\lambda_{i}-$ соответственно:

$$
\lambda_{1}=\ln \frac{\varepsilon_{p}(\eta=0)}{\varepsilon_{p}(\eta=1)}-\text { коэффициент чувствительности пластичности к изменению схе- }
$$

мы напряженного состояния в области показателя $0 \leq \eta \leq 1$;

$$
\lambda_{2}=\ln \frac{\varepsilon_{p}(\eta=-1)}{\varepsilon_{p}(\eta=0)}-\text { коэффициент чувствительности пластичности к изменению }
$$

схемы напряженного состояния в области показателя $-1 \leq \eta \leq 0$. 
В нашем случае для выбранной стали $\varepsilon_{p}(\eta=-1)=1-$ сжатие, $\varepsilon_{p}(\eta=0)=0,65$ сдвиг, $\lambda_{1}=0,431$, тогда $\varepsilon_{p}=1,37$. Подставив $\varepsilon_{p}=1,37$ в формулу (6), получим $W_{\text {уд }}=4187 \mathrm{MПа.}$

Энергию деформации рассчитываем по формуле (7), причем $h_{i}$ определяем из условия постоянства объема $V_{0}=V_{\text {def }}$ или:

$$
\frac{\pi d_{0}^{2}}{4} h_{0}=\frac{\pi d_{i}^{2}}{4} h_{i}
$$

откуда

$$
h_{i}=\frac{d_{0}^{2} \cdot h_{0}}{d_{i}^{2}}=\frac{7,62 \cdot 5,5}{11,34}=2,44 \mathrm{MM} .
$$

Тогда:

$$
V_{d e f}=\frac{\pi d_{i}^{2}}{4} h_{i}=\frac{\pi \cdot 11,43^{2}}{4} \cdot 2,44=250 \mathrm{Mm}^{3} .
$$

Энергия деформации:

$$
W_{\text {def }}=W_{y \partial} V_{\text {def }}=4187 \cdot 0,25=1046,75 \text { Дж. }
$$

С целью оценки использованного ресурса пластичности в процессе выдавливания полости в упрочняющемся металле (рис. 1) необходимо сформировать карту материала, которая, кроме кривой течения, включает также диаграмму пластичности.

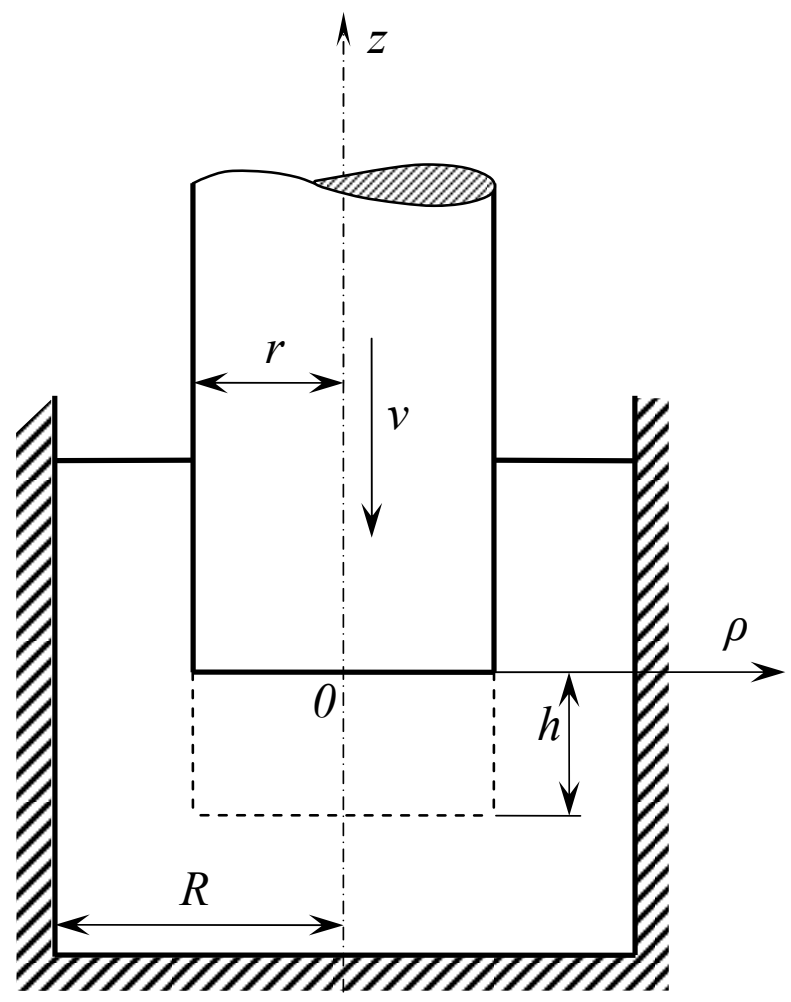

Рис. 1. Расчетная схема процесса обратного выдавливания 
На рис. 2 показана диаграмма пластичности стали 4X13. Экспериментальные данные для построения диаграммы пластичности получены в работе [1]. Диаграмма построена по результатам испытаний стали $4 \mathrm{X} 13$ на сжатие $\left(\varepsilon_{r}(\eta=-1)=3.3\right)$, сдвиг $\left(\varepsilon_{r}(\eta=0)=0.84\right)$ и аппроксимирована уравнением (8).

В процессе вдавливания пуансона в упрочняющийся металл изменяется показатель $\eta$ и степень деформации $\varepsilon_{i}$. Траектория $\varepsilon_{i}=f(\eta)$ называется «путь деформирования» частиц материала в опасной области деформируемой заготовки. Расчет использованного ресурса пластичности $\psi$ согласно критерию [5]:

$$
\psi=\int_{0}^{e_{u}^{\prime}}\left(1+0,2 \operatorname{arctg} \frac{d \eta}{d e_{u}}\right) \frac{e_{u}^{0,2 \operatorname{arctg} \frac{d \eta}{d e_{u}}} d e_{u}}{\left[e_{p\left(e_{u}\right)}\right]^{1+0,2 \operatorname{arctg} \frac{d \eta}{d e_{u}}}} \leq 1
$$

дает возможность оценивать предельное деформирование в процессе выдавливания полостей в упрочняющемся металле.

Заметим, что путь деформирования частиц материала, как указано в работе [6], слабо зависит от свойств материала, поэтому, зная карту материала, можно оценивать предельное формоизменение.

На рис. 1 показаны геометрические размеры пуансона радиуса $r$ и деформируемой заготовки радиуса $R$. Расчет использованного ресурса пластичности $\psi$ стали 40Х13 в процессе обратного выдавливания согласно критерию (9) дает значение $\psi=0,9$.

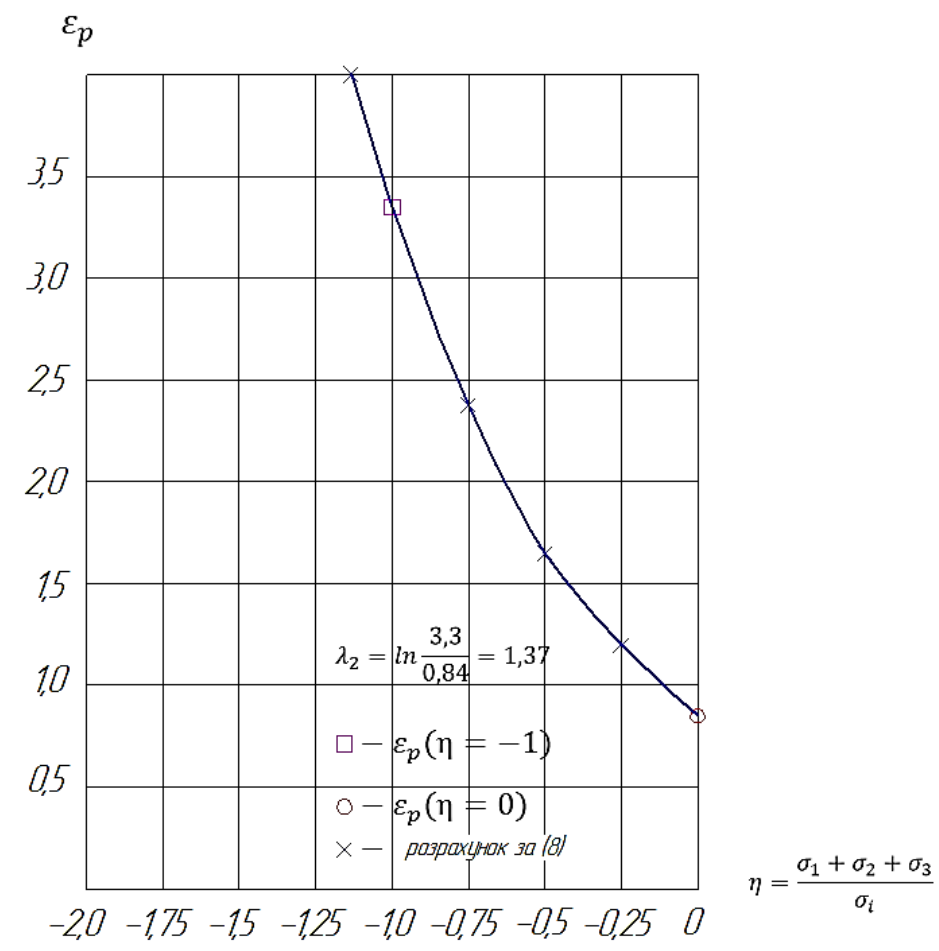

Рис. 2. Диаграмма пластичности стали 40X13

Для случая снятия упрочнения материала заготовки, деформированной по схеме (см. рис. 1) путем термической обработки (например, отжиг) необходимо знать степени предварительной холодной деформации, обеспечивающей минимальный рост зерна. Известно, что размеры зерен при рекристаллизации в условиях отжига связаны с распределением пластической деформации в процессе внедрения пуансона в пластически деформируемую среду. Зависимость размеров зерен от интенсивности деформации представлена диаграммой рекристаллизации [3]. Диаграмма рекристаллизации представляет зависимость размера зерна от степени холодной пластической деформации. 
При внедрении пуансона с наконечниками различной геометрии различными обжатиями возникают пластические деформации. Технологический процесс должен обеспечивать распределение деформации, при которых исключается неконтролируемый рост зерна после термической обработки.

\section{ВЫВОДЫ}

Разработан метод оценки деформационной способности сталей повышенной прочности с высокой энергопоглощаемостью при ударной нагрузке твердым пуансоном, позволяющий определять энергию деформирования. Данный метод основан на реконструкции карты материала по физико-механическим характеристикам.

Определена зависимость, позволяющая рассчитать коэффициент сопротивления упрочняющейся среды стали повышенной прочности с высокой энергопоглощаемостью.

Дана оценка исследованного ресурса пластичности при внедрении пуансона в деформируемую заготовку.

Показана возможность назначения технологических режимов в операциях внедрения пуансона, обеспечивающих прочность изделий путем снижения размеров зерен после термической обработки.

\section{СПИСОК ИСПОЛЬЗОВАННОЙ ЛИТЕРАТУРЫ}

1. Огородников В.А., Киселев В.Б., Сивак И.О. Энергия. Деформации. Разрушение (задачи автотехнической экспертизы): монография. Вінниця: УНІВЕРСУМ. 2005. 204 с.

2. Григорян В.А., Белобородько А.Н., Дорохов Н.С. и др. Частные вопросы конечной баллистики. Москва: МГТУ им. Н. Э. Баумана. 2006. 591 с. ISBN 5-7038-2798-1.

3. Дель Г.Д. Технологическая механика. Москва: Машиностроение. 1978. 174 с.

4. Огородников В.А. Оценка деформируемости металлов при обработке давлением. Киев: Вища школа. Головное изд-во. $1983.175 \mathrm{c.}$

5. Дель Г.Д., Огородников В.А., Нахайчук В.Г. Критерий деформируемости металлов при обработке давлением. Известия ВУЗов. Машиностроение. 1975. 4. С. 135-140.

6. Огородніков В.А., Кириця І.Ю., Перлов В.С. Механіка процесів холодного пластичного деформування вісесиметричніх заготовок з глухим отвором: монографія. Вінниця: ВНТУ. 2015. 164 с.

\section{REFERENCES}

1.Ogorodnikov V.A., Kiselev V.B., Sivak I.O. Energy. Deformations. Destruction (tasks of automotive technical expertise): monograph. Vinnitsya: Universum. 2005, 204 p. (in Russian).

2.Grigoryan V.A., Beloborodko A.N., Dorokhov N.S., et al. Private questions of final ballistics. Moscow: MSTU N.E. Bauman. 2006, 591 p. ISBN 5-7038-2798-1. (in Russian).

3.Del G.D. Technological mechanics. Moscow: Mechanical Engineering. 1978, 174 p. (in Russian).

4.Ogorodnikov V.A. Evaluation of the deformability of metals during pressure processing. Kyiv: Higher school. 1983, 175 p. (in Russian).

5.Del G.D., Ogorodnikov V.A., Nakhaychuk V.G. Criterion for deformability of metals during pressure Izvestiya. Engineering. 1975, 4, pp. 135-140. (in Russian).

6. Ogorodnikov V.A., Kiritsya I.Yu., Perlov V.C. Mechanics of cold plastic deformation of axisymmetric workpieces with a blind opening: monograph. Vinnitsa: VNTU. 2015, 164 p. (in Ukrainian).

Огородников В. А. - д-р техн. наук, проф., зав. кафедрой ВНТУ;

E-mail: va.ogorodnikow@gmail.com;

Архипова Т. Ф. $\quad$ - канд. техн. наук, доц. ВНТУ.

E-mail: tfarhipova@gmail.com

ВНТУ - Винницкий национальный технический университет, г. Винница. 